\title{
Airplane Crew Support Implementation Basing on Ellipsoidal Model of the Closed "Pilot-Airplane" Ergatic System
}

\author{
Veniamin N. Evdokimenkov ${ }^{1}$, Roman $\mathrm{Kim}^{1}$, Mikhail N. Krasilshchikov ${ }^{1}$ and Oleg N. Korsun ${ }^{2, *}$ \\ ${ }^{1}$ Moscow Aviation Institute (National Research University), 125080 Moscow, Russia \\ ${ }^{2}$ FGUP “GosNIIAS”, 125319 Moscow, Russia
}

\begin{abstract}
The paper discusses the problem of closed-loop ergatic "pilot - airplane" system monitoring for the purposes of flight safety. The new approach is proposed, based on the confidential ellipsoidal model, which parameters are estimated by flight data processing. The ellipsoidal model of ergatic "pilot - airplane" system enables to detect system emergency operation mode and to identify the source of such emergency mode. The operability of the proposed approach is confirmed by the experimental data processing.
\end{abstract}

\section{Introduction}

Analysis of aviation accidents shows, that they are mainly caused by the following factors:

- on-board failures of the airplane and its equipment ( $22 \%$ of aviation accidents);

- pilot's misunderstanding of the current flight situation and resulting pilot's inadequate response $(20 \%$ of aviation accidents);

- pilot's control errors, that is the manipulating the control levers in unfit mode ( $18 \%$ aviation accidents).

All the above mentioned factors refer to the general ergatic "pilot-airplane" system, that is the closed-loop system which includes pilot, plane, engines, control system, cockpit's indication etc. It is clear that these factors are crucial from the viewpoint of flight safety, so the mathematic model of this ergatic system may be of a great value. The paper proposes a new model for onitoring the state of the closed-loop ergatic system "pilot-airplane" based on the concept of multidimensional confidence ellipsoids. This ellipsoidal model of ergatic system is a intellectual kernel of the suggested approach. This model provides an opportunity to predict flight safety threat. The parameters of the ellipsoidal model of ergatic "pilot-airplane" system are estimated using the flight data.

\section{Ellipsoidal model of closed ergatic "pilot-airplane" system and its use for flight safety threat identification}

Let us assume, that ergatic system "pilot-airplane" state vector in every time instant $t$ has a block structure $\mathbf{Z}^{\mathrm{T}}(t)=\left(\mathbf{X}^{\mathrm{T}}(t), \mathbf{U}^{\mathrm{T}}(t)\right)$, where vector $\mathbf{X}(t)$ includes parameters of the aircraft motion, and vector $\mathbf{U}(t)$ includes pilot's generated controls such as pitch control stick, throttle lever, pedals, etc. All the components of these vectors are measured and saved by the on-board system of flight data measurement and registration.

Let's consider a typical flight task, for example, the landing of the aircraft. Let us assume, that in the multidimensional space, corresponding to the state vector $\mathbf{Z}^{\mathrm{T}}(t)=\left(\mathbf{X}^{\mathrm{T}}(t), \mathbf{U}^{\mathrm{T}}(t)\right)$, there is a subspace $E(t)$ which principal property is the following:

If in any instant of time $t$ ergatic system state vector $\mathbf{Z}(t)$ belongs to a subspace $E(t)$ the considered flight task may be completed successfully with great probability $\beta$, which is close to 1 . Let us denote this subspace $E(t)$ as accessibility subspace [1-5].

We can also use the approximation of this accessibility subspace in the multidimensional space in the form of parallelepiped as well as sphere or correlation ellipsoid with a given probability measure [4].

Let us consider the approximation of accessibility subspace $E(t)$ as the correlation ellipsoid $E^{\beta}(t)$ with given probability measure $\beta$ :

$$
E^{\beta}(t)=\left\{\mathbf{Z}(t):\left(\mathbf{Z}(t)-\mathbf{m}_{Z}(t)\right)^{T} \mathbf{K}_{Z}^{-1}\left(\mathbf{Z}(t)-\mathbf{m}_{Z}(t)\right) \leq R_{Z}(\beta)\right\},
$$

Where $\mathbf{m}_{z}(t)$ is mathematical expectation of "pilotairplane" system state vector at instant $t$ for all the flight paths corresponding to successful flight task completion; $\mathbf{K}_{Z}(t)$ is covariation matrix of "pilot-airplane" system state vector at instant $t$ for all the flight paths corresponding to successful flight task completion; $R_{Z}(\beta)$ is specific dimension of correlation ellipsoid, which correspond to confidential probability $\beta$.

Let us further refer the $E^{\beta}(t)(1)$ as a confidential ellipsoid.

The above defined confidential ellipsoid $E^{\beta}(t)$ approximates the accessibility subspace $E(t)$ and enables to consider statistical relation between airplane flight

Corresponding author: marmottoduo@yandex.ru

C) The Authors, published by EDP Sciences. This is an open access article distributed under the terms of the Creative Commons Attribution License 4.0 (http://creativecommons.org/licenses/by/4.0/). 
parameters and pilot's control signals. It would make possible to carry out the identification of flight safety threats. Algorithms for estimating confidential ellipsoid $E^{\beta}(t)$ parameters using the flight data are detailed in [5].

The confidential ellipsoid $E^{\beta}(t)$ being formed, it is possible for every instant $t$ of the flight path to estimate the process of piloting from the viewpoint of the flight safety. For this purpose let's assume that measurement errors of vector $\mathbf{Z}(t)$ components are negligible and may be used instead of this vector $\mathbf{Z}(t)$. If this assumption is not valid it's always possible to use Kalman filtering or any other statistical estimators [2]. For systematic errors methods like [6] schould be applied. So, the flight safety analysis of ergatic "pilot-airplane" system is reduced to checking the condition:

$$
\mathbf{Z}(t) \in E^{\beta}(t)
$$

If for an an arbitrary time instant $t$ flight data meets the condition (2), the ergatic "pilot-airplane" system is operating normally, i.e. any threats to flight safety are absent with guaranteeing probability $\beta$. In the opposite, the break of the condition (2) should be treated as an indication of emergency operation mode in the closedlooped "pilot-airplane" contour. So, the condition (2) is integral indicator of "pilot-airplane" mode of operation. However, condition (2) does not enable to identify the cause of emergency situation.

As it was said above, these causes may be divided into three groups:

- on-board failures of the airplane and its equipment;

- pilot's misunderstanding of the current flight situation; - pilot's control errors, i.e. the insufficient pilot's control activities coordination.

To generate formal criteria for emergency causes identification, it's necessary to consider the formulas (1) and (2) in a detailed way. First we are to note that the correlation ellipsoid (1) and the condition (2) are equivalent to the following inequation:

$$
\left(\mathbf{Z}(t)-\mathbf{m}_{Z}(t)\right)^{T} \mathbf{K}_{Z}^{-1}\left(\mathbf{Z}(t)-\mathbf{m}_{Z}(t)\right) \leq R_{Z}(\beta) .
$$

Considering block structure of the vector $\mathbf{Z}(t)$, the correlation matrix $\mathbf{K}_{Z}(t)$ may also be presented in blocks:

$$
\mathbf{K}_{Z}=\left[\begin{array}{ll}
\mathbf{K}_{\mathrm{XX}} & \mathbf{K}_{\mathrm{XU}} \\
\mathbf{K}_{\mathrm{UX}} & \mathbf{K}_{\mathrm{UU}}
\end{array}\right] .
$$

Let's designate the inverse matrix $\mathbf{K}_{z}^{-1}$ as follows:

$$
\mathbf{K}_{\mathrm{z}}^{-1}=\left[\begin{array}{ll}
\mathbf{P}_{\mathrm{XX}} & \mathbf{P}_{\mathrm{XU}} \\
\mathbf{P}_{\mathrm{UX}} & \mathbf{P}_{\mathrm{UU}}
\end{array}\right]
$$

Using evident relation

$$
\mathbf{K}_{\mathrm{Z}} \mathbf{K}_{\mathrm{Z}}^{-1}=\mathbf{E},
$$

where $\mathbf{E}$ is the unit matrix, we can write analytic expressions for elements of inverse matrix $\mathbf{K}_{\mathrm{z}}^{-1}$ :

$$
\begin{aligned}
& \mathbf{P}_{\mathrm{XX}}=\left(\mathbf{K}_{\mathrm{XX}}-\mathbf{K}_{\mathrm{XU}} \mathbf{K}_{\mathrm{UU}}^{-1} \mathbf{K}_{\mathrm{UX}}\right)^{-1}, \\
& \mathbf{P}_{\mathrm{XU}}=-\mathbf{K}_{\mathrm{XX}}^{-1} \mathbf{K}_{\mathrm{XU}}\left(\mathbf{K}_{\mathrm{UU}}-\mathbf{K}_{\mathrm{UX}} \mathbf{K}_{\mathrm{XX}}^{-1} \mathbf{K}_{\mathrm{XU}}\right)^{-1}, \\
& \mathbf{P}_{\mathrm{UU}}=\left(\mathbf{K}_{\mathrm{UU}}-\mathbf{K}_{\mathrm{UX}} \mathbf{K}_{\mathrm{XX}}^{-1} \mathbf{K}_{\mathrm{XU}}\right)^{-1} .
\end{aligned}
$$

The inequation (3) may be represented in the following form:

$$
\begin{aligned}
& \left(\mathbf{X}(t)-\mathbf{m}_{\mathrm{X}}(\mathrm{t})\right)^{\mathrm{T}} \mathbf{P}_{X X}\left(\mathbf{X}(t)-\mathbf{m}_{\mathrm{X}}(\mathrm{t})\right)+ \\
& 2\left(\mathbf{X}(t)-\mathbf{m}_{\mathrm{X}}(\mathrm{t})\right)^{\mathrm{T}} \mathbf{P}_{\mathrm{XU}}\left(\mathbf{U}(t)-\mathbf{m}_{\mathrm{U}}(\mathrm{t})\right)+ \\
& \left(\mathbf{U}(t)-\mathbf{m}_{\mathrm{U}}(t)\right)^{\mathrm{T}} \mathbf{P}_{\mathrm{UU}}\left(\mathbf{U}(t)-\mathbf{m}_{\mathrm{U}}(\mathrm{t})\right) \leq R_{Z}(\beta)
\end{aligned}
$$

Let's involve the following designation:

$$
\begin{aligned}
& R_{\mathrm{XX}}(t)=\left(\mathbf{X}(t)-\mathbf{m}_{\mathrm{X}}(t)\right)^{\mathrm{T}} \mathbf{P}_{\mathrm{XX}}\left(\mathbf{X}(t)-\mathbf{m}_{\mathrm{X}}(t)\right) \\
& \left.R_{\mathrm{XU}}(t)=2\left(\mathbf{X}(t)-\mathbf{m}_{\mathrm{X}}(t)\right)\right)^{\mathrm{T}} \mathbf{P}_{\mathrm{XU}}\left(\mathbf{U}(t)-\mathbf{m}_{\mathrm{U}}(t)\right) \\
& \left.R_{\mathrm{UU}}(t)=\left(\mathbf{U}(t)-\mathbf{m}_{\mathrm{U}}(t)\right)\right)^{\mathrm{T}} \mathbf{P}_{\mathrm{UU}}\left(\mathbf{U}(t)-\mathbf{m}_{\mathrm{U}}(t)\right)
\end{aligned}
$$

As a result, condition (3) may be presented in the form:

$$
R_{\mathrm{XX}}(t)+R_{\mathrm{XU}}(t)+R_{\mathrm{UU}}(t) \leq R_{\mathrm{Z}}(\beta)
$$

It's clear that in place of the inequation (10) it's possible to consider the following system of inequations:

$$
\begin{aligned}
& R_{\mathrm{XX}}(t) \leq R_{\mathrm{xx}}^{\alpha}(t), \\
& R_{\mathrm{XU}}(t) \leq R_{\mathrm{xU}}^{\alpha}(t), \\
& R_{\mathrm{UU}}(t) \leq R_{\mathrm{UU}}^{\alpha}(t),
\end{aligned}
$$

where parameters $R_{\mathrm{xx}}^{\alpha}(t), R_{\mathrm{xu}}^{\alpha}(t), R_{\mathrm{uu}}^{\alpha}(t)$ correspond to guaranteeing probability $\alpha=\sqrt[4]{\beta}$.

The estimates for $R_{\mathrm{xx}}^{\alpha}(t), R_{\mathrm{xu}}^{\alpha}(t), R_{\mathrm{uU}}^{\alpha}(t)$ are determined by processing the flight data $\mathbf{X}^{j}(t), \mathbf{U}^{j}(t), j=1, \ldots, N$ according to the algorithms presented in [7]. Let's discuss conditions (11) in a detailed way.

Condition

$R_{\mathrm{XX}}(t)=\left(\mathbf{X}(t)-\mathbf{m}_{\mathrm{X}}(t)\right)^{\mathrm{T}} \mathbf{P}_{X X}\left(\mathbf{X}(t)-\mathbf{m}_{\mathrm{X}}(t)\right) \leq R_{\mathrm{XX}}^{\alpha}(t)$

describes for an arbitrary time instant $t$ statistical relation between components of the airplane state vector $\mathbf{X}(t)$. This vector includes flight parameters which depend on the state of the airplane, engines and on-board equipment. If the inequation (12) is not satisfied, the airplane and the on-board facilities are in emergency mode of operation. In other words, the condition (12) is a state indicator for the technical part of the ergatic "pilotairplane" system.

Condition

$$
R_{\mathrm{XU}}(t)=2\left(\mathbf{X}(t)-\mathbf{m}_{\mathrm{X}}(t)\right)^{\mathrm{T}} \mathbf{P}_{\mathrm{XU}}\left(\mathbf{U}(t)-\mathbf{m}_{\mathrm{U}}(t)\right) \leq R_{\mathrm{XU}}^{\alpha}(t)
$$

reflects statistical relation between airplane state parameters $\mathbf{X}(t)$ and control parameters $\mathbf{U}(t)$. This relation depends on the degree of compatibility between the pilot control activities and the current state of the airplane. If the inequality (13) is not satisfied it may be a proof of insufficient pilot's attentiveness as well as of 
pilot's ignoring some information signals. Moreover, analysis of signals, which break the condition (13) enables to localize information signal ignored by the pilot. In this case a warning should be generated in order to concentrate the pilot's attention.

Inequation

$$
R_{\mathrm{UU}}(t)=\left(\mathbf{U}(t)-\mathbf{m}_{\mathrm{U}}(t)\right)^{\mathrm{T}} \mathbf{P}_{\mathrm{UU}}\left(\mathbf{U}(t)-\mathbf{m}_{\mathrm{U}}(t)\right) \leq R_{\mathrm{UU}}^{\alpha}(t)
$$

reflects statistical relation between various pilot's controls (throttle lever, pitch control stick, etc.) If for an arbitrary time instant $t$ the inequality (14) is not satisfied it may be a proof of the insufficient pilot's control activities coordination. Moreover, analysis of condition (14) enables to identify the clumsy control signals. In this case the generation of a warning is also recommended.

\section{Some examples of "pilot-airplane" system state estimation, based on ellipsoidal model}

The practical use of the described above ellipsoidal model for identification of flight safety threats requires statistical estimates of the model parameters $\mathbf{m}_{\mathrm{X}}(t)$, $\mathbf{m}_{\mathrm{U}}(t), \mathbf{P}_{\mathrm{XX}}(t), \mathbf{P}_{\mathrm{XU}}(t), \mathbf{P}_{\mathrm{UU}}(t), R_{\mathrm{xx}}^{\alpha}(t), R_{\mathrm{xu}}^{\alpha}(t), R_{\mathrm{UU}}^{\alpha}(t)$. It means that for every piloting task a great amount of flight data is needed. The investigations, carried out by the authors, confirm that the proposed approach is valid when the considerable part of the data is generated in the piloting simulation facilities. Naturally, it's obligatory that the flight data and the simulation correspond to the same type of the airplane, the same piloting task and the same pilot.

As an example let us consider such an important piloting task as the landing. Let us assume that the necessary amount of the flight data is obtained from real flights, simulation or the both. Since the landing path and the airspeed of the aircraft are subject of a strong regulation, there is a stable relation between time $t$ and the distance $l$ from the airplain and the runway center. It means that in expressions (1)-(14) for ellipsoidal model of closed-loup ergatic "pilot-airplane" system it is possible to use distance $l$ instead of time $t$.

So, let's suppose that the flight data is presented in the form of realizations $\mathbf{X}^{j}(l), \mathbf{U}^{j}(l), j=1, \ldots, N$, which are the result of landing flight mode, obtained by actual flight and simulation. Let's assume that the estimates for $\mathbf{m}_{\mathrm{X}}(l), \mathbf{m}_{\mathrm{U}}(l), \mathbf{P}_{\mathrm{XX}}(l), \mathbf{P}_{\mathrm{XU}}(l), \mathbf{P}_{\mathrm{UU}}(l)$ are calculated, using standard statistical approach for samples $\mathbf{X}^{j}(l)$, $\mathbf{U}^{j}(l), j=1, \ldots, N$. Corresponding algorithms are detailed in [7].

The ellipsoidal model, generated on the basis of $\mathbf{m}_{\mathrm{X}}(l)$, $\mathbf{m}_{\mathrm{U}}(l), \mathbf{P}_{\mathrm{XX}}(l), \mathbf{P}_{\mathrm{XU}}(l), \mathbf{P}_{\mathrm{UU}}(l), R_{\mathrm{xx}}^{\alpha}(l), R_{\mathrm{xu}}^{\alpha}(l), R_{\mathrm{uU}}^{\alpha}(l)$ may be used for identification of flight safety threats.

In order to obtain the detailed identification parameters

$$
\begin{aligned}
& \left.R_{\mathrm{XU}}(l)=2\left(\mathbf{X}(l)-\mathbf{m}_{\mathrm{X}}(l)\right)^{\mathrm{T}} \mathbf{P}_{\mathrm{XU}}(\mathbf{U}(l))-\mathbf{m}_{\mathrm{U}}(l)\right) \\
& R_{\mathrm{UU}}(l)=\left(\mathbf{U}(l)-\mathbf{m}_{\mathrm{U}}(l)\right)^{\mathrm{T}} \mathbf{P}_{\mathrm{UU}}\left(\mathbf{U}(l)-\mathbf{m}_{\mathrm{U}}(l)\right)
\end{aligned}
$$

are to be calculated, using actual values of $\mathrm{X}(l)$ and $\mathrm{U}(l)$ vectors, where $l$ is distance from center of runway.

The obtained values $R_{X U}(l)$ and $R_{U U}(l)$ for an arbitrary value $l$ should be compared with corresponding values of $R_{\mathrm{xu}}^{\alpha}(l), R_{\mathrm{vu}}^{\alpha}(l)$. The results are presented on Fig. 1-4.

Fig. 1 shows $R_{X U}(l)$ for landing modes, which have been completed according to all landing accuracy requirements. Fig. 2 shows corresponding relations for landing modes, in which the landing accuracy requirements were broken due to insufficient pilot's attentiveness. Fig. 3 presents $R_{U U}(l)$ for landing modes, which have been completed according to all landing accuracy requirements. Fig. 4 gives corresponding relations for landing modes, which have been completed with the break of landing accuracy requirements as the result of insufficient coordination of pilot's control activities.

Analysis shows, that for landing modes, completed according to landing accuracy requirements (Fig.1, 3) at any arbitrary instant of landing the following conditions are met:

$$
\begin{aligned}
& R_{\mathrm{XU}}(l)<R_{\mathrm{xU}}^{\alpha}(l), \\
& R_{\mathrm{UU}}(l)<R_{\mathrm{UU}}^{\alpha}(l) .
\end{aligned}
$$
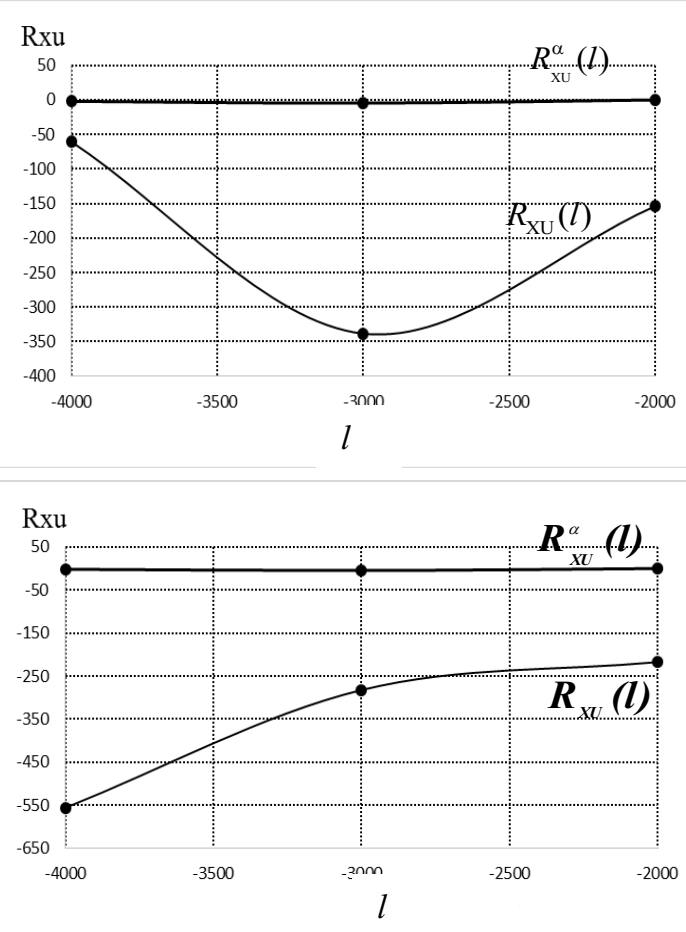

Fig. 1. $R_{\mathrm{XU}}(l), R_{\mathrm{XU}}^{a}(l)$ for landing modes, satisfying the landing accuracy requirements

In contrast, for landing modes, completed with the break of landing accuracy requirements at some instants of landing process corresponding conditions are not met (Fig.2, 4):

$$
\begin{aligned}
& R_{\mathrm{XU}}(l)>R_{\mathrm{xu}}^{\alpha}(l), \\
& R_{\mathrm{UU}}(l)>R_{\mathrm{UU}}^{\alpha}(l) .
\end{aligned}
$$




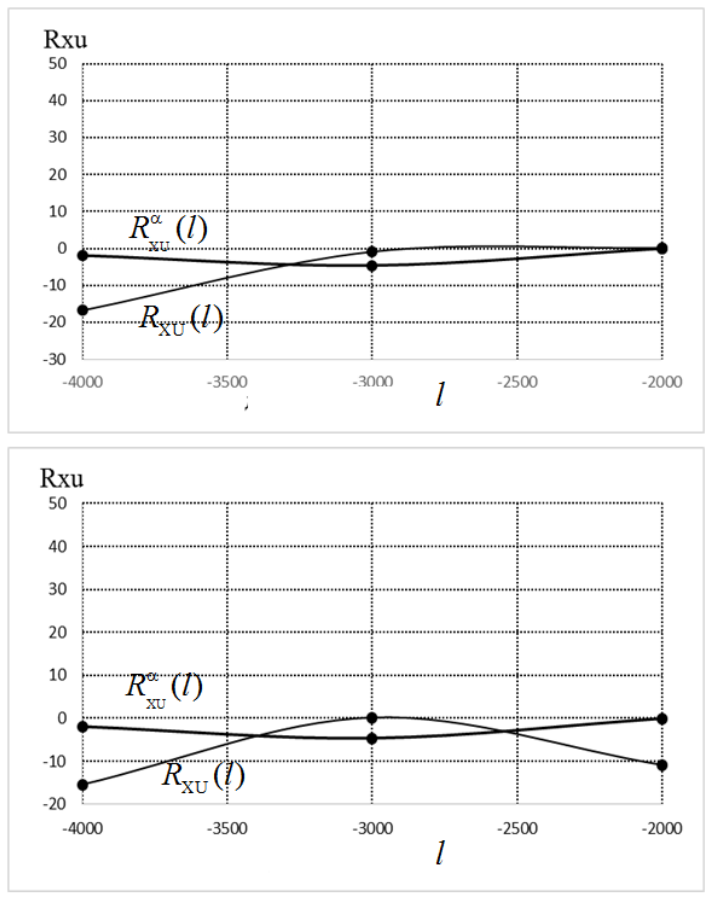

Fig. $2 R_{\mathrm{XU}}(l), R_{\mathrm{XU}}^{a}(l)$ for landing modes, breaking landing accuracy requirements
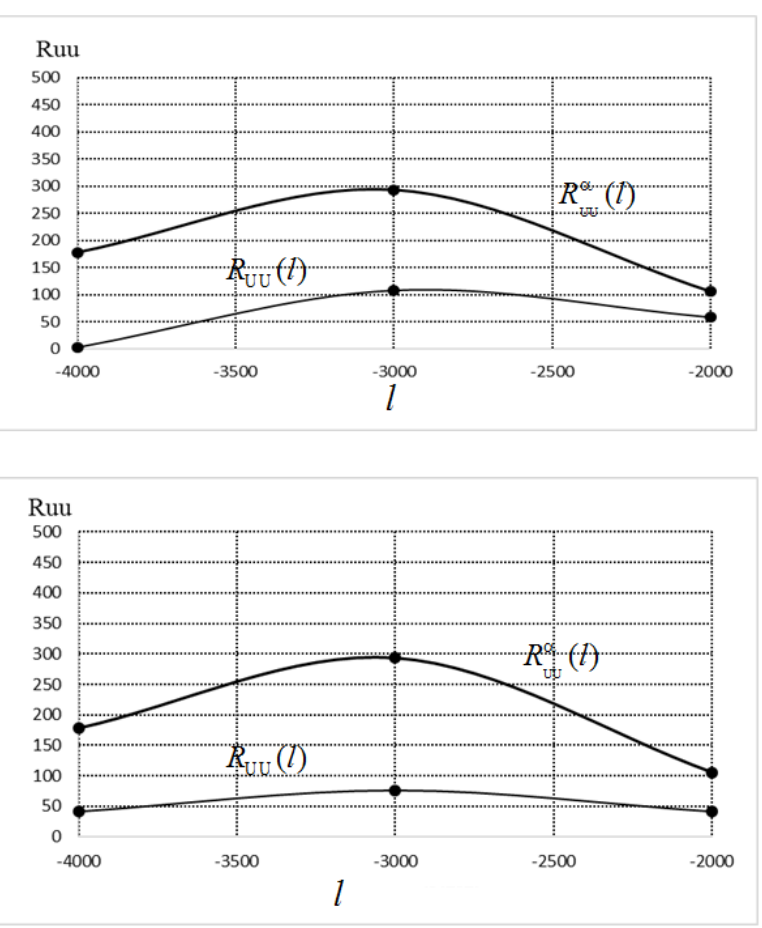

Fig. 3. $R_{\mathrm{UU}}(l), R_{\mathrm{UU}}^{\alpha}(l)$ relations for landing modes, satisfying the landing accuracy requirements
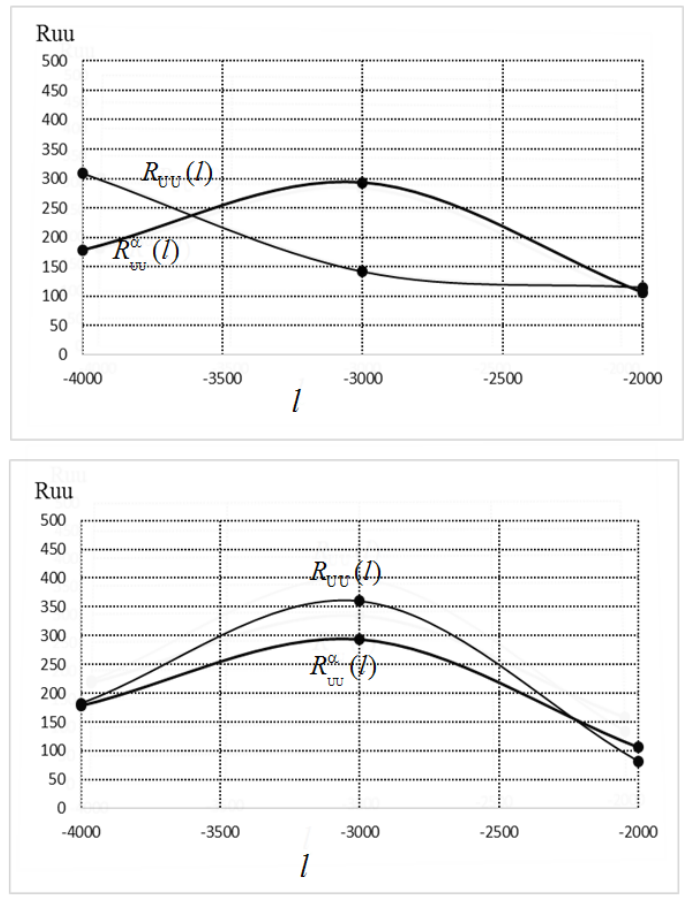

Fig. 4. Relations $R_{\mathrm{UU}}(l), R_{\mathrm{UU}}^{\alpha}(l)$ relations for landing modes, breaking the landing accuracy requirements

\section{Conclusion}

Thus, the processing of the experimental results confirm that the proposed ellipsoidal model of ergatic "pilotairplane" system provides the detection of emergency modes of operation and also the identification of flight safety threats.

\section{References}

1. F.L. Chernousko, Estimation of dynamic system phase state (Moscow, Nauka, 1988).

2. I.A. Boguslavskiy. Journal of Computer and Systems Sciences International, 35(4), 511-521 (1996)

3. V.V. Malyshev, A.I. Kibzun, Analysis and synthesis of high accurate verifying vehicle control (Moscow, Mashinostroenie, 1987)

4. V.V. Malyshev, M.N. Krasilshchikov. V.I. Karlov, Optimization of observation and control of flying vehicles (Moscow, Mashinostroenie, 1989)

5. V.N. Evdokimenko., R.V. Kim, M.N. Krasil'shchikov, G.G. Sebryakov, Journal of Computer and Systems Sciences International, 54(4), 609-620 (2015)

6. O.N. Korsun, S.V. Nikolaev, S.G. Pushkov, Journal of computer and systems sciences international, 55(3), 446-457 (2016)

7. M.N. Krasilshchikov, V.N. Evdokimenkov, D.A. Bazlev. Individually-adapted on-board systems of flying vehicles technical state monitoring and crew support (Moscow, MAI, 2011). 